\title{
A Preliminary Investigation of the Aerodynamic Characteristics of a Lifting Body in Ground Proximity
}

\author{
Jenny Holt ${ }^{1}$, Kevin Garry ${ }^{2}$ and Tony Smith $^{3}$ \\ Applied Aerodynamics Group. Cranfield University BEDFORD MK43 OAL UK
}

\begin{abstract}
The use of cambered hull shapes in the next generation of lighter-than-air vehicles to enhance aerodynamic performance, together with optimized take-off manoeuvre profiles, will require a more detailed understanding of ground proximity effects for such aircraft. A series of sub-scale wind tunnel tests at $\operatorname{Re}=1.4 \times 10^{6}$ on a $6: 1$ prolate spheroid are used to identify potential changes in aerodynamic lift, drag and pitching moment coefficients that are likely to be experienced on the vehicle hull in isolation when in close ground proximity. The experimental data is supported by a preliminary assessment of surface pressure changes using a high order panel method (PANAIR) and RANS CFD simulations to assess the flow structure. The effect of ground proximity, most evident when non-dimensional ground clearance $(h / c)<0.3$, is to reduce lift coefficient, increase drag coefficient and increase the body pitching moment coefficient.
\end{abstract}

\section{Nomenclature}

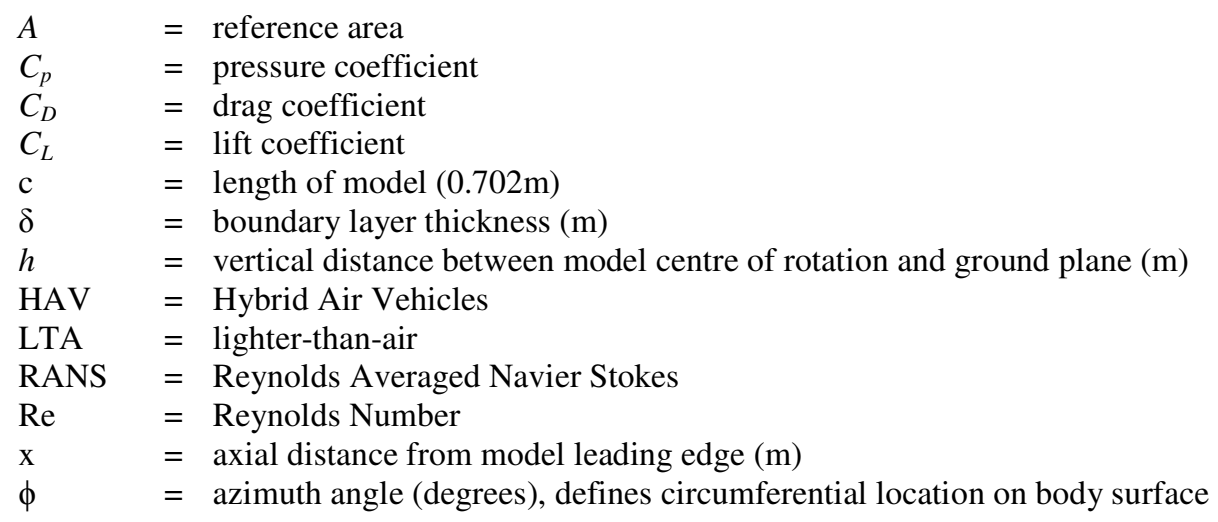

\section{Background and Introduction}

$\mathrm{T}$ There is current interest in the use of lifting body configurations to enhance the performance of lighter-than-air (LTA) vehicles. Hybrid LTA configurations, such as the HAV Airlander 10 shown in Figure 1, exploit geometric camber in the hull shape to create aerodynamic lift. In such configurations conventional buoyancy, produced by the lifting gas, typically produces between 60 and $100 \%$ of the vehicle lift, while lift from the body shape can account for $0-40 \%$ and the vectored propulsor units can produce nominally $25 \%$. As a consequence the flight envelope of hybrid configurations can be expanded beyond that of conventional LTA vehicles. This paper addresses some of the aerodynamic performance implications that arise specifically during take-off and landing phases when the vehicle can be considered to be in close ground proximity.

The take-off technique for Hybrid LTA configurations is generally considered to fall into one of three broad categories:

\footnotetext{
${ }^{1}$ Lecturer, Applied Aerodynamics Group. SATM Cranfield University UK MAIAA

${ }^{2}$ Professor of Experimental Aerodynamics, Applied Aerodynamics Group. SATM Cranfield University UK MAIAA

${ }^{3}$ Aerospace Dynamics MSc Candidate (Academic Year 2014-2015), Cranfield University UK

1

American Institute of Aeronautics and Astronautics
} 
(i) Rolling Take-Off. Power is applied to initiate a ground-run. At a given velocity the vehicle incidence is increased, normally by the use of conventional horizontal control surfaces, and the vehicle climbs with the aid of body-generated aerodynamic lift.

(ii) Up-Ship Take-Off. The vehicle becomes airborne from a standing position at a horizontal attitude under buoyancy lift. Power is applied at the appropriate manoeuvre safety altitude to initiate forward flight.

(iii) Vectored Take-Off. Power is applied at the desired vector angle and the vehicle lifts off whilst accelerating forwards. Appropriate control surface deflections together with changes in vector angle can be used to optimize the body lift component when the flight speed is sufficient, typically $20-25 \mathrm{kts}$.

In the case of a Rolling take-off and, to a slightly lesser extent, a Vectored take-off, there is the potential for significant aerodynamic interaction with the ground. There is relatively little data available to support prediction of aerodynamic forces and moments for such configurations during take-off whilst in ground proximity, see for example; Silverstein \& Gulick [3] and Calkins [4]. This paper outlines the results from a series of preliminary wind tunnel experiments which were designed to investigate the changes in both the flow field and aerodynamic forces and moments on a simple symmetric body in proximity with a ground plane. The experimental wind tunnel programme was optimised using a series of numerical simulations comprising both a high-order panel method and RANS CFD on an idealized axisymmetric body. The primary aim was to identify regions of the take-off flight envelope which may require similar simulations to be undertaken in further studies on a proposed, more complex, hybrid LTA vehicle.

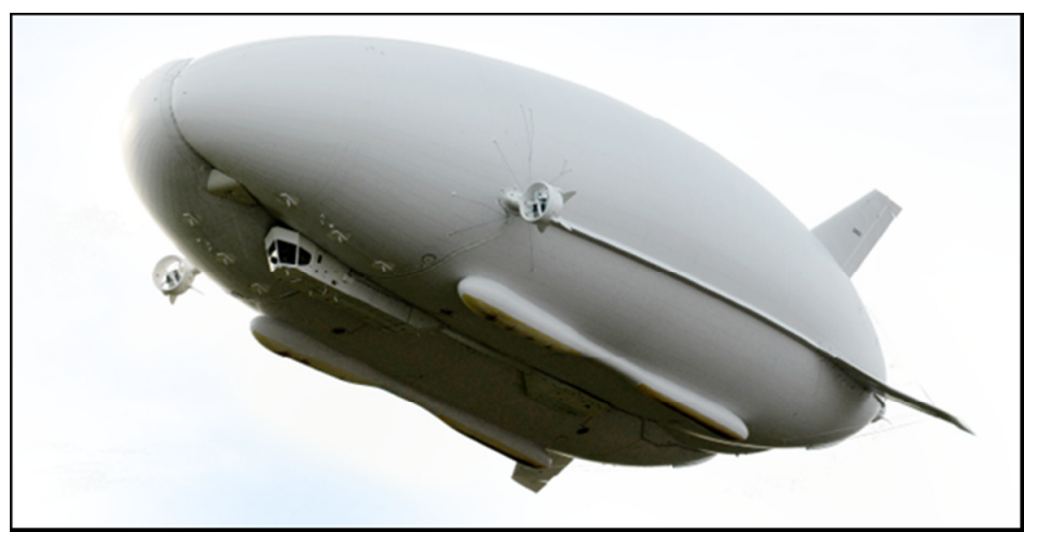

Figure 1. The Hybrid Air Vehicles (HAV) Airlander 10 configuration which exhibits the characteristic lifting body profile used to enhance aerodynamic performance, [1]

\section{Methodology}

\section{(a) The preliminary PANAIR model}

The simplified geometry chosen for this preliminary investigation was a prolate spheroid with a lateral to longitudinal axis ratio of $6: 1$ and chord (c) of $0.702 \mathrm{~m}$. This shape has been shown to exhibit similar characteristics to classic mono-hull airship configurations, see for example Jones et.al. [2], and was considered suitable for an assessment of both the experimental techniques and the potential effectiveness of numerical simulations.

Design of the experimental test programme was supported by estimates of surface pressure distribution using a high-order panel method - PANAIR [5], [7], [8] and [9]. This approach had previously been shown by Tseng and Llorens [6] to give a good approximation to the pressure distribution around simple LTA vehicle hull shapes, out of ground effect and at low angle of incidence, typically less than 10 degrees.

PANAIR operates by approximating the body geometry as a series of discrete panels on which singularities of unknown strength are defined. The body surface must therefore be organized into a structured mesh and the resulting node coordinates input in a specific NASA Langley Wireframe Geometry Standard (LaWGS) format. A 
bespoke MATLAB routine was written to facilitate this process, details are given by Smith [11]. PANAIR assumes the flow to be irrotatonal and inviscid, as a result viscous boundary layer separation is not modeled and the resulting data needs to be treated with caution in areas where these effects are significant.

An important aspect of the PANAIR computational domain is the construction of a representation of the wake within the domain. In this case, estimates of the location of boundary layer separation, for a given angle of incidence, were taken from experimental data for the 6:1 prolate spheroid. This data was assumed to represent the start of the wake region and was used as an input in the bespoke MATLAB routine. The resulting wake representation is formed from separation streamlines which extend rearwards, parallel to the free stream, and is modeled as a series of discrete panels, as used for the body.

Predictions of surface pressure coefficient using PANAIR show reasonable agreement with experimental data, upstream of boundary layer separation, particularly for the body lower surface ( $\phi=180$ degrees), see Figure 2.
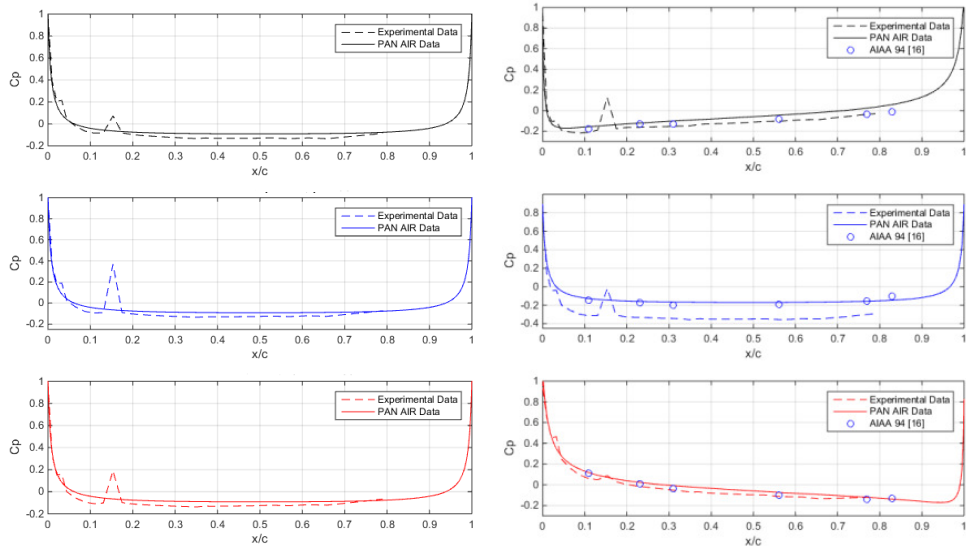

(a) $\alpha=0$ degrees

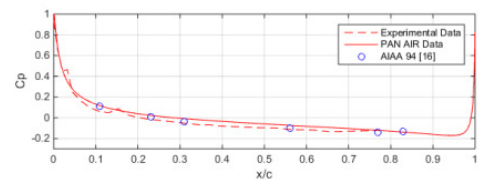

(b) $\alpha=10$ degrees
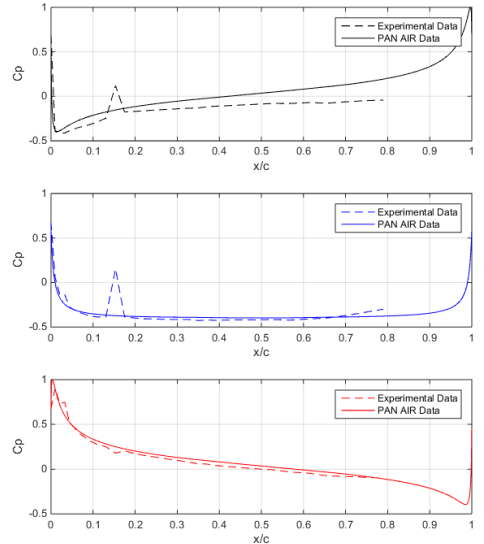

(c) $\alpha=20$ degrees

Figure 2. Comparison between measured and predicted surface static pressure distributions for the 6:1 prolate spheroid, out of ground -effect, at three model azimuth angles: $\phi=0$ degrees (upper surface), $\phi=90$ degrees (side) and $\phi=180$ degrees (lower surface), corresponding to the top, centre and bottom rows of the Figure respectively.

\section{(b) The wind tunnel model and experimental arrangement}

Wind tunnel measurements on a sub-scale model were undertaken in the 'Weybridge' open working section, closed return facility at Cranfield University. The contraction nozzle exit diameter is $1.067 \mathrm{~m}$ and the working section dynamic pressure (nominally $30 \mathrm{~m} / \mathrm{s}$ for the tests outlined in this paper) is monitored using static pressure rings located in the wind tunnel nozzle and the settling chamber, together with a prior calibration of the empty test section. Corrections to allow for working section blockage were implemented as outlined by Garner et al [12].

The 6:1 prolate spheroid model, length $0.702 \mathrm{~m}$, reference area $0.0113 \mathrm{~m}^{2}$ includes an internal, 6 -component, force/moment balance. It is supported from a single overhead strut which allows the model to pivot about a point on its longitudinal axis to vary the angle of incidence. The model pivot point is coincident with the wind tunnel working section horizontal and axial centre, see Figure 3. Model boundary layer transition is fixed using a serrated tape positioned around the body circumference, at $\mathrm{x} / \mathrm{c}=0.15$. The nominal test Reynolds number is $1.4 \times 10^{6}$, based on model length (c).

A full width adjustable ground board $(1.42 \mathrm{~m}$ long) was used to simulate the presence of the ground in the open working section. The ground clearance $(\mathrm{h})$ was varied by raising/lowering the ground board relative to the (fixed) model. Note that as a result the blockage correction is a function of model ground clearance. 

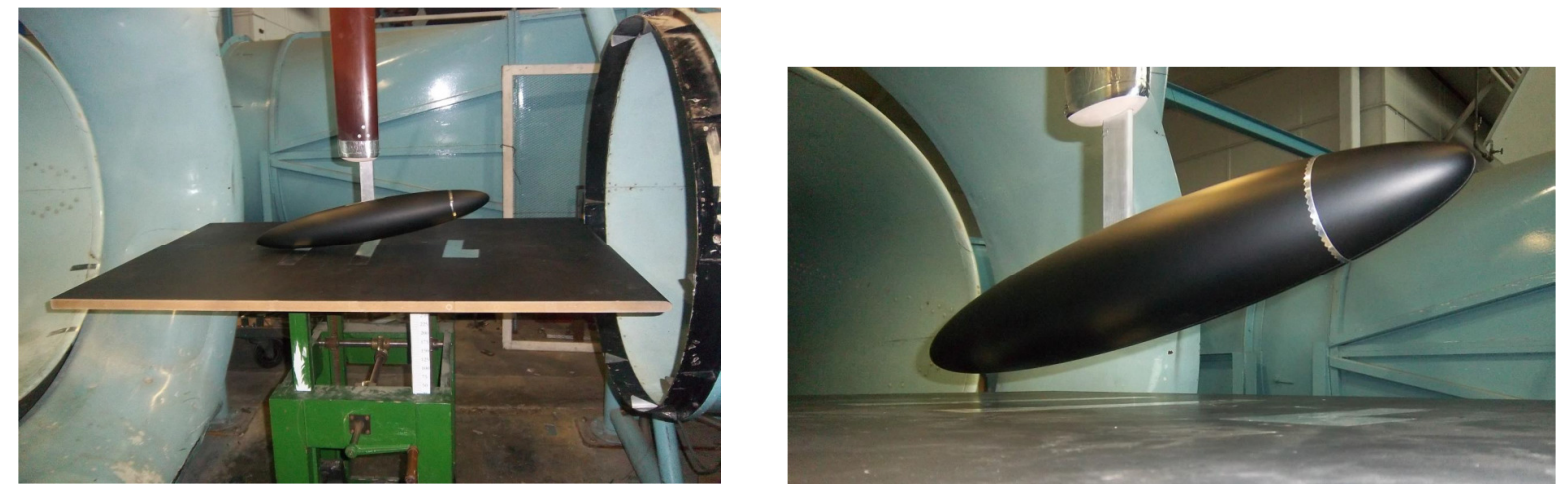

Figure 3. The prolate spheroid model mounted from an overhead strut in the open jet wind tunnel above an adjustable ground board (free stream flow direction is from right to left)

\section{(c) The RANS CFD Simulation}

A RANS CFD simulation using Ansys FLUENT was carried out to aid understanding of the flow field changes as a result of ground proximity effects. An initial out-of-ground-effect simulation used previously published data, [13],[14] and [16], to explore the effectiveness of various turbulence models and the near-wall k- $\omega$ SST model was adopted for subsequent simulations with ground proximity. An unstructured hexa-core volume computational mesh was constructed using the ICEM package such that values of the non-dimensional wall unit (y+) were in the range $1<\mathrm{y}+<\delta$. Preliminary mesh dependency studies showed that a domain with 7.2 million cells appeared to capture the key features of the flow field; specific details are given in Smith [11].

\section{Results and Discussion}

\section{(a) Preliminary assessment using PANAIR derived pressure data}

PANAIR was used to conduct a preliminary assessment of the effect of ground proximity on the pressure distribution around the prolate spheroid, primarily to aid the design of the wind tunnel test and associated instrumentation.

It is seen that, as expected, the largest effect of ground proximity appears in the pressure data on the body surface closest to the ground. The longitudinal variation of surface static pressure coefficient $(\mathrm{Cp})$ along the lower surface of the body (corresponding to azimuth angle, $\phi=180$ degrees) with ground clearance and body angle of incidence $(\alpha)$ is shown in Figure 4. The data show that the difference in the pressure distribution relative to that seen out of ground-effect, is negligible when $\mathrm{h} / \mathrm{c}>0.3$. At incidence angles likely to be attained during the take-off manoeuvre, changes in the pressure distribution were primarily towards the rear of the body, at axial locations corresponding to $\mathrm{x} / \mathrm{c}>0.5$. It should be recognised that the PANAIR simulation does not reflect boundary layer separation and the magnitude of predicted $\mathrm{Cp}$ changes in this region should therefore only be used as an indication of possible ground proximity effects. 


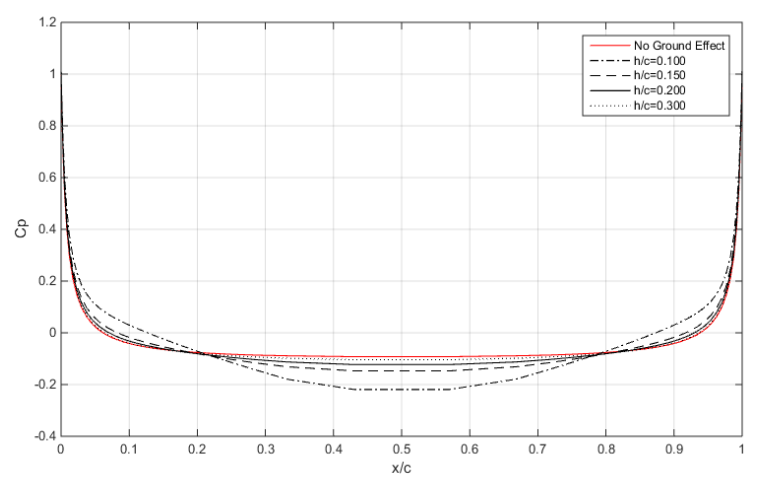

(a) $\alpha=0$ degrees

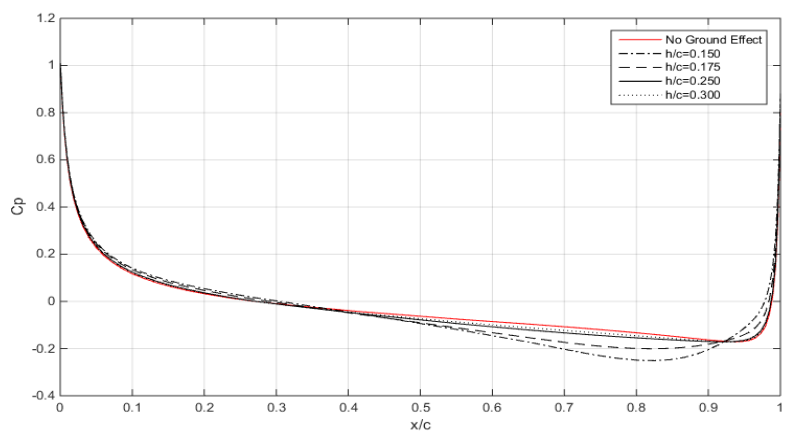

(b) $\alpha=10$ degrees

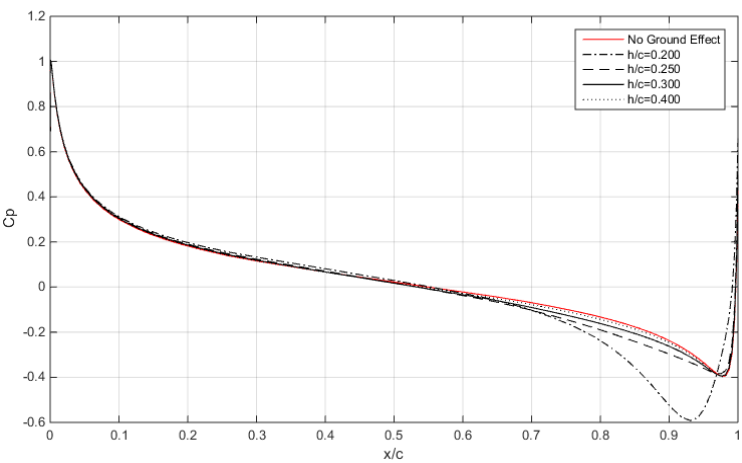

(c) $\alpha=20$ degrees

Figure 4. Longitudinal pressure coefficient distribution prediction along the body lower surface centre line $(\phi=180$ degrees) using PANAIR for the 6:1 prolate spheroid in ground effect at incidence angles 0,10 and 20 degrees.

\section{(b) wind tunnel aerodynamic load measurements}

As a consequence of the PANAIR predictions of surface static pressure, the experimental arrangement was designed to investigate aerodynamic loads on the prolate spheroid model for model ground clearance in the range $0.043<\mathrm{h} / \mathrm{c}<0.499$ and angles of incidence from zero to 20 degrees.

The influence of ground proximity on the body lift coefficient $\left(\mathrm{C}_{\mathrm{L}}\right)$, shown in Figure 5, illustrates a reduction in $\mathrm{C}_{\mathrm{L}}$ with ground proximity that is most apparent when the ground clearance $(\mathrm{h} / \mathrm{c})<0.2$. This reduction is weakly dependent on body incidence (resulting in little measurable change in lift-curve slope) and is accompanied by an increase in drag coefficient $\left(C_{D}\right)$ with reducing ground clearance. The resulting effect on the body lift-to-drag ratio is shown in Figure 6. The change in lift coefficient with reducing ground clearance is attributed to the reduction in pressure seen towards the rear of the body as the flow accelerates between the body and the ground. The difference 
in measured lift coefficient in and out of ground proximity $\left(\mathrm{C}_{\mathrm{L}}-\mathrm{C}_{\mathrm{Linf}}\right)$ for various body incidence angles, is shown in Figure 9. At the higher incidence angles tested and away from close ground proximity $(0.12<\mathrm{h} / \mathrm{c}<0.4)$ there is an increase in measured lift coefficient which is assumed to originate from an effective angle of incidence increase.

The effect of reducing ground clearance on pitching moment coefficient $\left(\mathrm{C}_{M}\right)$, shown in Figure 7 , is to increase $\mathrm{C}_{\mathrm{M}}$ and this increase is seen to be greater at higher body incidence. As a consequence the gradient of pitching moment coefficient with incidence $\left(\mathrm{dC}_{\mathrm{M}} / \mathrm{d \alpha}\right)$, increases with reducing ground clearance. The difference in measured pitching moment coefficient in and out of ground proximity $\left(\mathrm{C}_{M}-\mathrm{C}_{\text {Minf }}\right)$ for various body incidence angles, is shown in Figure 10. It can be seen that, whilst the magnitude of the measured pitching moment change is nominally the same at each incidence tested, the sensitivity of the pitching moment coefficient to ground proximity is greater at higher body incidence.

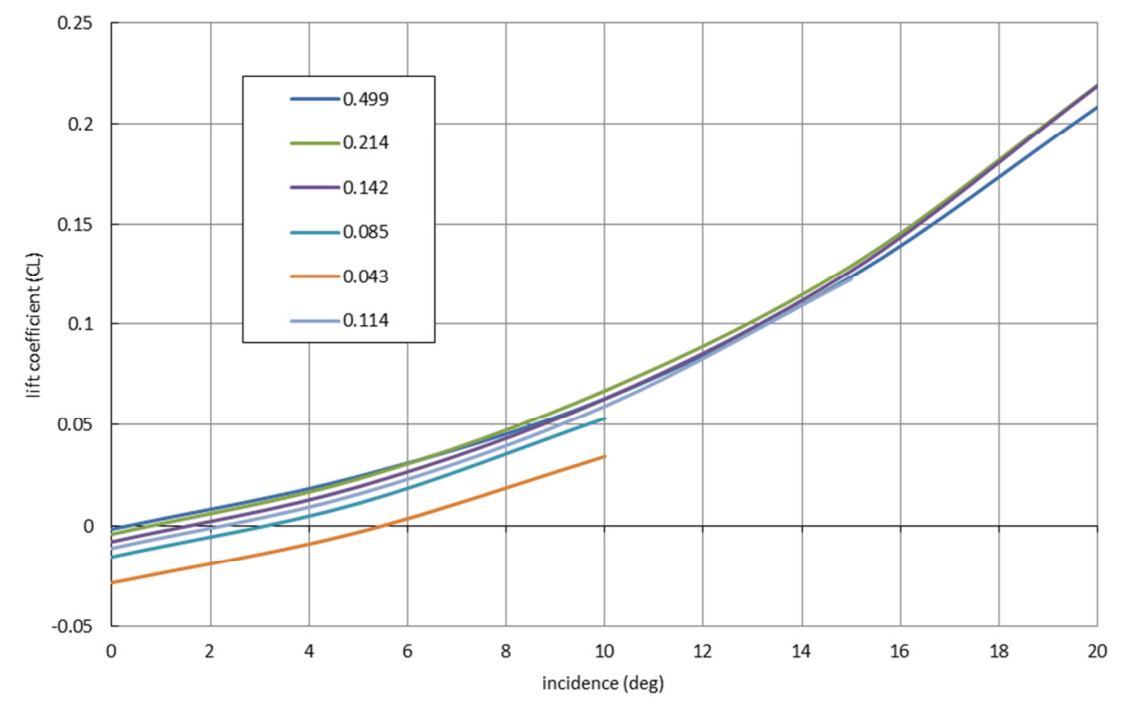

Figure 5. Variation of lift coefficient $\left(C_{L}\right)$ for the prolate spheroid wind tunnel model at $R e=1.4 \times 10^{6}$ with incidence $(\alpha)$ for a range of model ground clearance, $0.114<h / c<0.499$.

(note the limitation in permissible model incidence with reducing ground clearance).

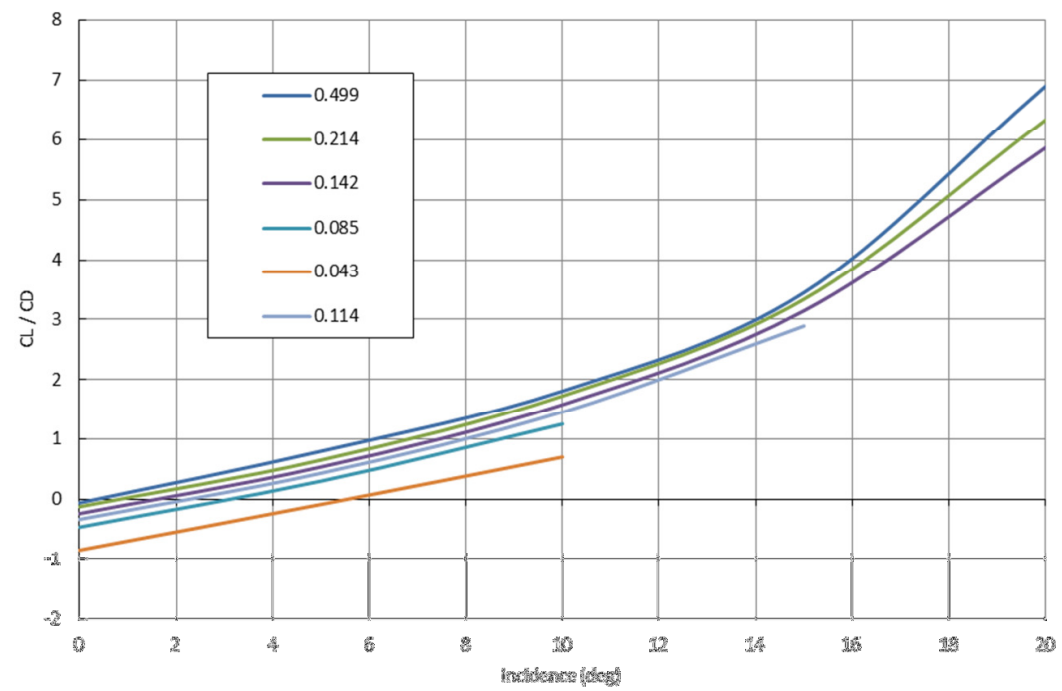

Figure 6. Variation of lift-to-drag ratio $\left(C_{L} / C_{D}\right)$ for the prolate spheroid wind tunnel model at $R e=1.4 \times 10^{6}$ with incidence (a) for a range of model ground clearance, $0.114<h / c<0.499$ 


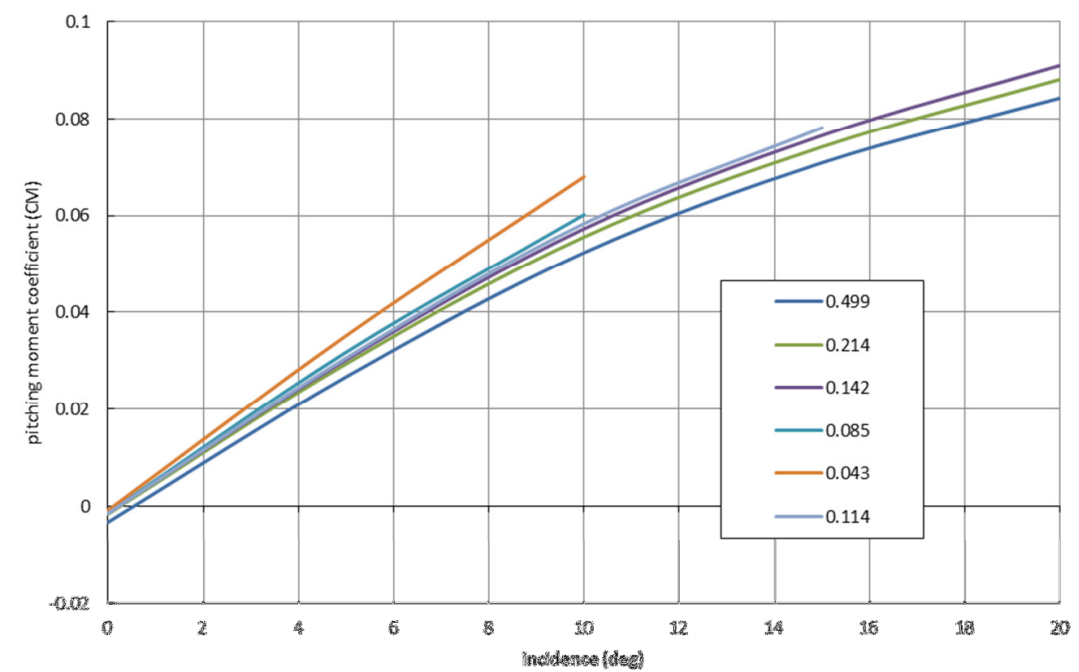

Figure 7. Variation of pitching moment coefficient $\left(C_{m}\right)$ for the prolate spheroid wind tunnel model at $R e=1.4 \times 10^{6}$ with incidence ( $\alpha$ ) for a range of model ground clearance, $0.114<h / c<0.499$

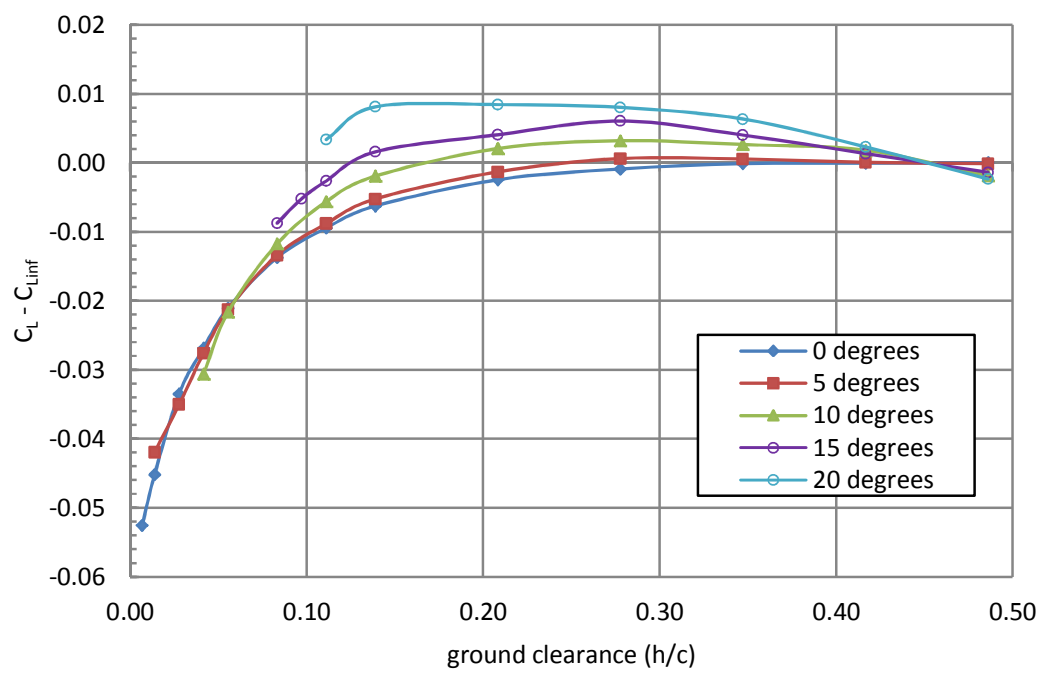

Figure 9. Change in lift coefficient $\left(C_{L}\right)$ due to ground proximity compared to the out-of-ground-effect condition $\left(C_{\text {Linf }}\right)$ for the range of body incidence angle $(\alpha)$ tested.

American Institute of Aeronautics and Astronautics 


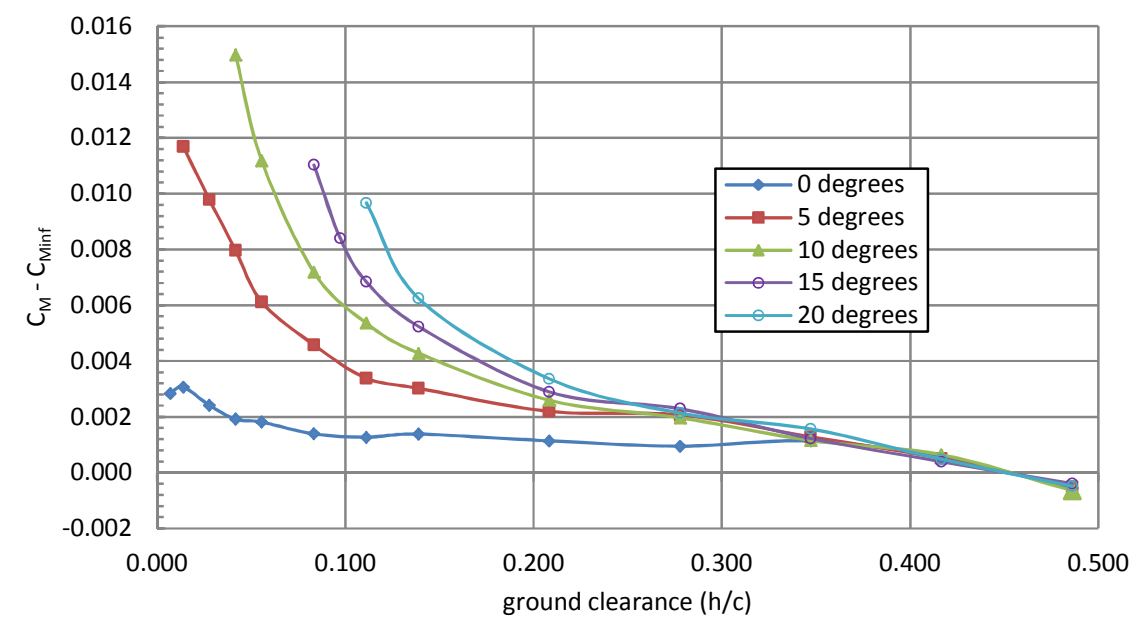

Figure 10. Change in pitching moment coefficient $\left(C_{M}\right)$ due to ground proximity compared to the out-of-ground-effect condition $\left(C_{\text {Minf }}\right)$ for the range of body incidence angle $(\alpha)$ tested.

\section{(c) RANS CFD simulation}

A series of numerical experiments were initially carried out to establish the optimum turbulence model and grid topology to adopt within the FLUENT RANS CFD simulation, complete details are given by Smith [11]. One of the methods used to make this assessment was to compare wall shear stress contours, predicted by the CFD simulation, with surface oil flow visualization on the wind tunnel model. This approach highlights the principle flow features that have been identified by previous researchers. Of specific interest in this investigation are flow separation lines, originating at the rear of the body and progressively moving forwards with increasing incidence angle. The flow structure in the region of this separation line is described by Wetzell and Simpson [13] who refer to both primary and secondary separation lines which can be located by inspection of both the computational solutions and flow visualization images, see Figure 12. Ground proximity is seen from the data obtained during these tests to influence the location of the primary separation line, but not the location of secondary separation, see Figure 13. It should be recognized that similar trends in the movement of the primary separation line are seen, out of ground proximity, due to changes in both: (i) the position of the boundary layer trip (relative to the body leading edge) and (ii) free stream Reynolds number, transition free. The practical significance of the sensitivity of the primary separation to these effects lies in the potential impact on the local flow orientation experienced by any rearbody mounted fins and control surfaces that will be used on the proposed LTA vehicle.

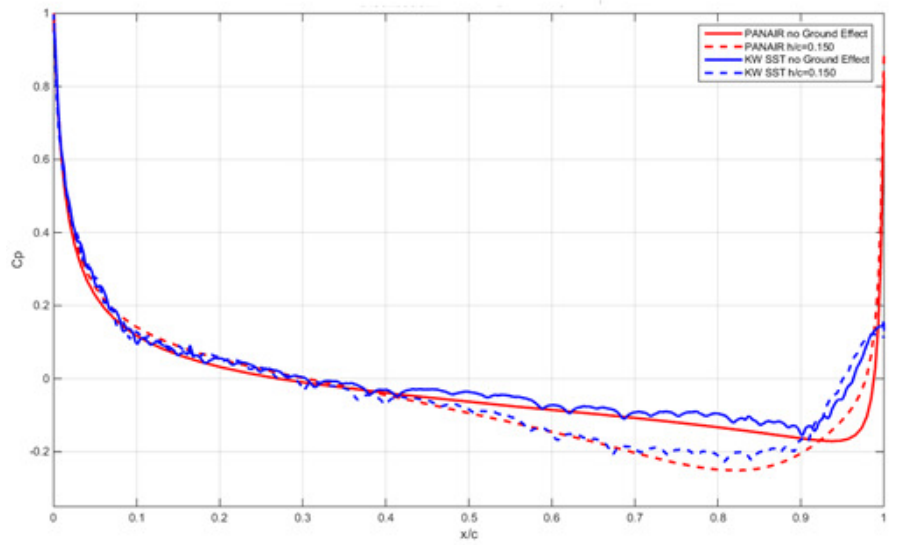

Figure 11. Comparison between the PANAIR and CFD predictions of longitudinal pressure distribution for the prolate spheroid lower surface ( $\phi=180$ degrees) in and out of ground effect $(h / c=0.15, \alpha=10$ degrees)

8

American Institute of Aeronautics and Astronautics 


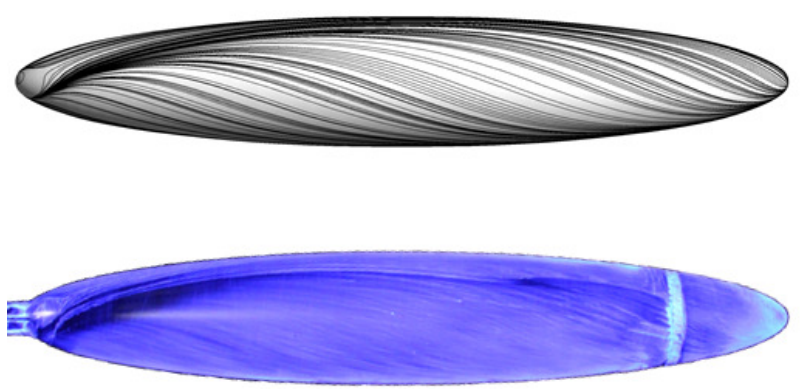

secondary separation

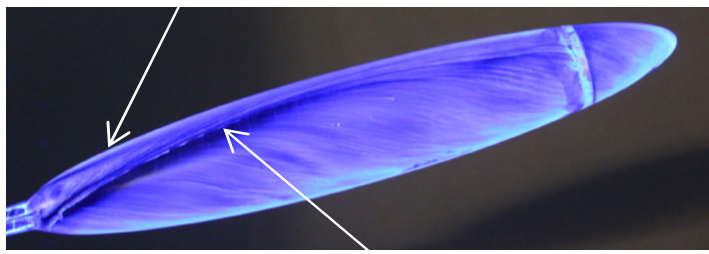

primary separation

Figure 12. Comparison between the experimental and CFD predictions of the prolate spheroid surface flow structure at $\alpha$ $=10$ degrees. (upper right image: Wall shear stress based streamlines from the FLUENT CFD simulation using the $k$ - $\omega$ SST turbulence model, lower right image: surface oil flow visualization, boundary layer transition strip at $x / c=0.15$ ).

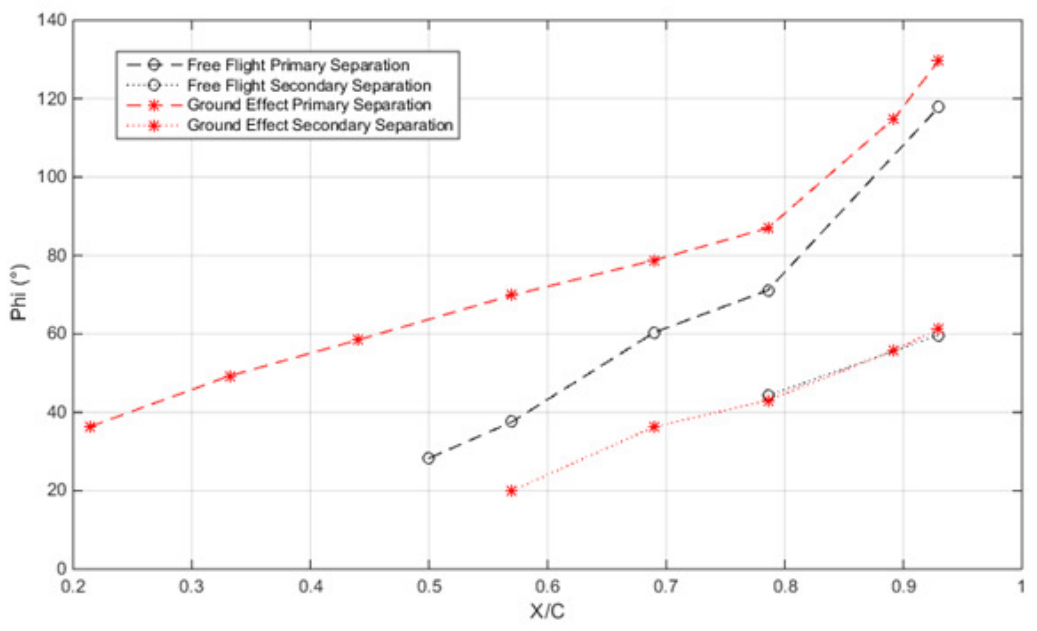

Figure 13. Summary of the influence of ground proximity on the positions of both the primary and secondary flow separation lines on the prolate spheroid ( $\alpha=10$ degrees)

\section{Conclusions}

A prolate spheroid, 6:1 axis ratio, is used to investigate the potential effect of ground proximity on the aerodynamic loads experienced by the envelope of lighter than air vehicles during the take-off ground run and initial stages of climb. Wind tunnel load measurements, at $\mathrm{Re}=1.4 \times 10^{6}$, with boundary layer transition fixed at $\mathrm{x} / \mathrm{c}=$ $15 \%$, show that the body lift coefficient $\left(\mathrm{C}_{\mathrm{L}}\right)$ decreases and drag coefficient $\left(\mathrm{C}_{\mathrm{D}}\right)$ increases with reducing ground clearance. The body pitching moment coefficient $\left(\mathrm{C}_{\mathrm{M}}\right)$ increases with reducing ground clearance and the gradient, $\delta \mathrm{CM} / \delta \alpha$ also increases. The effect of ground proximity $(\mathrm{h})$ is primarily experienced $\mathrm{when} h / \mathrm{c}<0.3$. A RANS CFD simulation using FLUENT shows good agreement with wind tunnel surface oil flow visualisation and is used to identify the changes in flow structure that occur as a result of ground proximity. Reducing ground clearance is seen to promote earlier crossflow separation on the body at incidence. 


\section{Acknowledgments}

The authors would like to recognize the financial support from the UK Technology Strategy Board (TSB) under the LOCATE programme - Collaborative industrial research into technology and manufacturing capability for a novel LOw CArbon aircraft using lighter than air Technology, Reference TS/L008696/1.

\section{References}

${ }^{1}$ http://www.hybridairvehicles.com, accessed 27th Oct 2015.

2 Jones, R and Williams, D.H. The distribution of pressure over the surface of airship model U.721, together with a comparison with the pressure over a spheroid. NACA Reports and Memoranda, No. 600, April 1919, p.304.

${ }^{3}$ Silverstein, A and Gulick, B.G. Ground handling forces on a $1 / 40^{\text {th }}$ scale model of the U.S. Airship Acron. NACA Report No566 (1936)

${ }^{4}$ Calkins, D.E. Feasibility study of a hybrid airship operating in ground effect. Jn. of Aircraft Vol.14 No.8 (1977)

${ }_{6}^{5}$ PDS (Public Domain Aeronautical Software) http://pdas.com

${ }^{6}$ Tseng, W. and Llorens, R. Application of the panel method to airships. Paper 83, 5th AIAA Lighter-Than Air Systems Conference, Anaheim, CA. U.S.A. (1983)

${ }^{7}$ Boeing, A5021 User's Manual - PAN AIR technology program for solving problems of potential flow about arbitrary configurations, Cage Code 81205, Document No. D6-54703.

${ }^{8}$ Sidwell, K.W., Baruah, P.K. and Bussoletti, J.E. PANAIR - a computer program for predicting subsonic or supersonic linear potential flows about arbitrary configurations using a higher order panel method. NASA Contractor Report 3252, Volume II - User's Manual (Version 1.0).

${ }^{9}$ Epton, M.A., Magnus, A.E. PANAIR - A Computer Program for Predicting Subsonic or supersonic linear potential flows about arbitrary configurations using a higher order panel method. NASA Contractor Report 3251, Volume I - Theory Document (Version 3.0)

${ }^{10}$ Schmidt, A. and Brunswig, H. The AC20.30 blended wing body configuration: development and current status 2006. Proc. 25th International Congress of the Aeronautical Sciences, Hambourg, Germany. (2006)

${ }^{11}$ Smith, T. An investigation of the influence of ground effect on a lifting bluff body. MSc Thesis. Cranfield University, UK (2015)

${ }^{12}$ Garner, H.C., Rogers, E.W.E., Acum, W.E.A. and Maskell, E.C. Subsonic Wind Tunnel Wall Corrections. NATO Advisory Group for Aerospace Research \& Development. AGARDoGraph 109 (1966)

${ }^{13}$ Wetzel, T.G. and Simpson, R.L. Unsteady Flow over a 6:1 Prolate Spheroid. Advanced Research Projects Agency, Office of Naval Research, Applied Hydrodynamics, VPI-AOE-232 (1996).

${ }^{14}$ Abbott, Ira H. Airship Model Tests in the Variable Density Wind Tunnel. NACA Report No.394 (1931).

${ }^{15}$ Kotapati-Apparao, R.B., Squires, K.D. and Forsythe, J.R. Prediction of a Prolate Spheroid Undergoing a Pitchup Maneuver, American Institute of Aeronautics and Astronautics, AIAA 2003-0269

${ }^{16}$ Hoang, N.T., Wetzel, T.G. and Simpson, R.L. Surface pressure measurements over a 6:1 prolate spheroid undergoing time-dependent maneuvers. American Institute of Aeronautics and Astronautics, AIAA-94-1908-CP (1994). 
2016-07-31

\section{Investigation of the aerodynamic} characteristics of a lifting body in ground proximity

Holt, Jenny C.

American Institute of Aeronautics and Astronautics

Jenny C. Holt, Kevin P. Garry, and Tony Smith. Investigation of the aerodynamic characteristics of a lifting body in ground proximity, Proceedings of the 34th AIAA Applied Aerodynamics Conference, Washington D.C., 13-17 June, 2016, USA. (AIAA 2016-3881)

http://dx.doi.org/10.2514/6.2016-3881

Downloaded from Cranfield Library Services E-Repository 